\title{
SiO maser astrometry of the red transient V838 Monocerotis ${ }^{\star}$
}

\author{
Gisela N. Ortiz-León ${ }^{1}$, Karl M. Menten ${ }^{1}$, Tomasz Kamiński ${ }^{2,3}$, Andreas Brunthaler ${ }^{1}$, \\ Mark J. Reid ${ }^{2}$, and Romuald Tylenda ${ }^{3}$
}

\author{
1 Max Planck Institut für Radioastronomie, Auf dem Hügel 69, 53121 Bonn, Germany \\ e-mail: gortiz@mpifr-bonn.mpg.de \\ 2 Center for Astrophysics | Harvard \& Smithsonian, 60 Garden Street, Cambridge, MA 02138, USA \\ 3 Nicolaus Copernicus Astronomical Center, Polish Academy of Sciences, Rabiańska 8, 87-100 Toruń, Poland
}

Received 11 February 2020 / Accepted 3 April 2020

\begin{abstract}
We present multiepoch observations with the Very Long Baseline Array of SiO maser emission in the $v=1, J=1-0$ transition at $43 \mathrm{GHz}$ from the remnant of the red nova V838 Mon. We modeled the positions of maser spots to derive a parallax of $0.166 \pm$ 0.060 mas. Combining this parallax with other distance information results in a distance of $5.6 \pm 0.5 \mathrm{kpc}$, which is in agreement with an independent geometric distance of $6.1 \pm 0.6 \mathrm{kpc}$ from modeling polarimetry images of V838 Mon's light echo. Combining these results, and including a weakly constraining Gaia parallax, yields a best estimate of distance of $5.9 \pm 0.4 \mathrm{kpc}$. The maser spots are located close to the peaks of continuum at $\sim 225 \mathrm{GHz}$ and $\mathrm{SiO} J=5-4$ thermal emission detected with the Atacama Large (sub)Millimeter Array. The proper motion of V838 Mon confirms its membership in a small open cluster in the Outer spiral arm of the Milky Way.
\end{abstract}

Key words. masers - techniques: interferometric - astrometry - supergiants - stars: distances - stars: individual: V838 Monocerotis

\section{Introduction}

V838 Mon is a remnant of a so-called red nova, which erupted in January 2002 (Munari et al. 2002a) and immediately came to prominence in the astronomical community and the public due to the spectacular images of a light echo taken with the Hubble Space Telescope (HST; Bond et al. 2003). This eruption probably resulted from a merger of two $\left(\simeq 8 M_{\odot}\right.$ and $\left.\simeq 0.3 M_{\odot}\right)$ stars (Tylenda \& Soker 2006), but other mechanisms have also been proposed. Munari et al. (2002b) found a composite optical (postoutburst) spectrum of V838 Mon consistent with a binary system of a cool giant and a hotter stellar companion (see also Wagner \& Starrfield 2002; Crause et al. 2003; Kamiński et al. 2009). Late in 2002, the cool giant resembled a late-M type star, while the hot companion appeared to be a B3 V star. The M-type star is naturally assumed to be the successor (remnant) of the outbursting star. More recent spectroscopic observations by Loebman et al. (2015) have classified the cool star as an L3 supergiant with an effective temperature of $\sim 2000-2200 \mathrm{~K}$. The separation between the two components was first estimated to be $28 \mathrm{AU}$ based on the assumption that V838 Mon is an eclipsing binary (Munari et al. 2007). This assumption has been proven to be incorrect and a system separation of $\sim 250 \mathrm{AU}$ was found instead (Tylenda et al. 2009).

Several scenarios have been proposed to explain the nature of the 2002 eruption (see the extended discussion in Tylenda \& Soker 2006), although the observational data illustrate that a stellar-merger event is the most viable explanation. In this scenario, the progenitor of V838 Mon was likely a triple or higher order system dominated by two B-type stars (the out-

\footnotetext{
* Fits images associated with Figs. 2-4 are only available at CDS via anonymous ftp to cdsarc.u-strasbg. fr (130.79.128.5) or via http://cdsarc.u-strasbg.fr/viz-bin/cat/J/A+A/638/A17
}

bursting star and the B3V companion; Tylenda \& Soker 2006). A low-mass companion might have entered in a highly eccentric orbit and finally merged with the primary.

Afşar \& Bond (2007) discovered that V838 Mon belongs to a sparse open cluster of young $(\lesssim 25 \mathrm{Myr})$ B-type stars. A diffuse molecular cloud within the echo region was revealed in $\mathrm{CO}$ single-dish radio observations by Kamiński et al. (2011). This cloud is thought to be interstellar in nature, consisting of material that remained after the formation of the cluster to which V838 Mon belongs.

The immediate surroundings of V838 Mon are rich in molecular gas. Optical and infrared spectroscopy reveal a multitude of atomic lines and molecular bands with very complex kinematics (Tylenda et al. 2011). Part of this neutral cool gas was lost either during or just before the merger, as in other Galactic red novae (Kamiński et al. 2018). Some of the absorption features of V838 Mon indicate the presence of ongoing mass loss from the coalesced star (Kamiński et al. 2009).

Searches for maser emission from oxygen-rich molecules $\left(\mathrm{SiO}, \mathrm{H}_{2} \mathrm{O}\right.$, and $\left.\mathrm{OH}\right)$ and hydrogen recombination lines have been conducted toward V838 Mon. Deguchi et al. (2005) made the first detection of $\mathrm{SiO}$ maser emission $(J=1-0, v=1$ and 2) at $43 \mathrm{GHz}$ with the Nobeyama $45 \mathrm{~m}$ telescope in 2005 , which was three years after the outburst. Claussen et al. (2007) reported follow-up $\mathrm{SiO}$ maser observations taken with the Very Large Array (VLA), the Green Bank Telescope (GBT), and the Very Long Baseline Array (VLBA). The maser emission observed with the VLA and the GBT showed variations in flux density superimposed on a rising trend (reaching a maximum of about $8 \mathrm{Jy}$ ) over $\mathrm{a} \approx 900$-day timescale, after which the flux started to decline. The VLA spectra showed one and two features in the $v=2$ and $v=1$ transitions, respectively, at LSR velocities near $54 \mathrm{~km} \mathrm{~s}^{-1}$. However, results from the VLBA observations have not been published. 
Table 1. VLBA observed epochs.

\begin{tabular}{lcccc}
\hline \hline ID BM464 & Observation date & JD & $\begin{array}{c}\text { Beam size } \\
(\mathrm{mas} \times \text { mas; deg })\end{array}$ & $\begin{array}{c}\mathrm{rms}^{-1} \\
\left(\mathrm{mJy} \mathrm{beam}^{-1}\right)\end{array}$ \\
\hline A & Oct 23, 2017 & 2458049.99042 & $0.65 \times 0.51 ;-45.0^{\circ}$ & 20 \\
B & Jan 13, 2018 & 2458131.76652 & $0.91 \times 0.54 ; 9.0^{\circ}$ & 17 \\
C & May 23, 2018 & 2458262.40884 & $0.74 \times 0.23 ;-16.2^{\circ}$ & 17 \\
D & Aug 27, 2018 & 2458358.64671 & $0.91 \times 0.43 ; 28.4^{\circ}$ & 27 \\
E & Nov 20, 2018 & 2458442.91462 & $0.68 \times 0.47 ; 20.8^{\circ}$ & 17 \\
F & Feb 25, 2019 & 2458539.64977 & $0.96 \times 0.44 ; 33.0^{\circ}$ & 21 \\
F1 & Mar 07, 2019 & 2458550.61973 & $0.75 \times 0.42 ; 16.6^{\circ}$ & 20 \\
\hline
\end{tabular}

More recently, the same $\mathrm{SiO}$ maser lines were observed using the Effelsberg $100 \mathrm{~m}$ telescope in October, 2013 and in January, 2017. It was found that between the two epochs the $v=2$ line declined from $\approx 0.3 \mathrm{Jy}$ to $<0.1 \mathrm{Jy}$, and the $v=1$ line declined from $\approx 2 \mathrm{Jy}$ to $\approx 1 \mathrm{Jy}$. This is consistent with the gradual decline of flux density which started in early 2006, as reported by Claussen et al. (2007).

The $\mathrm{SiO} v=1, J=2-1$ maser transition at $86 \mathrm{GHz}$ was also detected in this source with the $45 \mathrm{~m}$ telescope at Nobeyama (Deguchi et al. 2009) and more recently with the IRAM-30 m telescope (Kamiński et al. 2018). In addition, interferometric observations at millimeter/submillimeter wavelengths using the Submillimeter Array (SMA) revealed lines of $\mathrm{CO}, \mathrm{SiO}, \mathrm{SO}, \mathrm{SO}_{2}$, and $\mathrm{H}_{2} \mathrm{~S}$ covering a very broad velocity range ( $400 \mathrm{~km} \mathrm{~s}^{-1}$; Kamiński et al. 2018). These molecular transitions trace thermal emission from the merger ejecta and are orders of magnitude weaker in peak intensity than the $\mathrm{SiO}$ maser lines.

Originally the distance to V838 Mon was uncertain, with estimates ranging from 6 to $10 \mathrm{kpc}$ (Bond et al. 2003; Tylenda 2004; Munari et al. 2005). Based on the geometry of the light echo seen in polarimetric images taken with the HST, Sparks et al. (2008) derived a distance of $6.1 \pm 0.6 \mathrm{kpc}$. This value has been adopted by various authors when deriving the properties of the remnant.

In this paper, we report new VLBA observations of the $43 \mathrm{GHz} \mathrm{SiO}$ maser transitions toward V838 Mon. The phasereferencing technique was used to provide accurate astrometry. The VLBA observations are presented in Sect. 2 and their analysis in Sect. 3. The astrometric parameters and the distance and proper motion to the source are derived in Sect. 4. Over the course of the VLBA observations, the Gaia mission published its second data release (Gaia DR2; Gaia Collaboration 2018; Lindegren et al. 2018). We thus also compare our astrometry with that provided by Gaia. We discuss the parallax, position, and proper motions of V838 Mon in Sect. 5, as well as SiO thermal emission detected in new Atacama Large (sub)Millimeter Array (ALMA) observations. Finally, we present our conclusions in Sect. 6.

\section{Observations and data reduction}

\subsection{VLBA observations}

V838 Mon was observed with the VLBA for a total of 7 epochs between October, 2017 and March, 2019 (Table 1). The observations were taken at $43 \mathrm{GHz}$ with 4 intermediate frequency (IF) bands of $16 \mathrm{MHz}$ bandwidth. Except for the first epoch, two of these IFs were centered at the $v=1, J=1-0$ and $v=2, J=1-0$ $\mathrm{SiO}$ maser transitions, at $43122.080 \mathrm{MHz}$ and $42820.582 \mathrm{MHz}$, respectively (Müller et al. 2013), and correlated with a channel spacing of $31.25 \mathrm{KHz}\left(0.2 \mathrm{~km} \mathrm{~s}^{-1}\right)$. The first epoch only covered the $v=1, J=1-0$ transition. The quasars J0709-0255 $(\mathrm{RA}=07: 09: 45.0546, \mathrm{Dec}=-02: 55: 17.496)$ and J0656-0323 $(\mathrm{RA}=06: 56: 11.1206$, Dec $=-03: 23: 06.782)$, in J2000 coordinates, were observed as phase reference calibrators. Observations consisted of alternate scans on the target and one phasereference calibrator, switching sources every $\approx 20 \mathrm{~s}$. Blocks of about 30 min spent on calibrators distributed over a wide range of elevations were observed at $23.7 \mathrm{GHz}$ every $\approx 2 \mathrm{~h}$ during each $7 \mathrm{~h}$ observing run. These scans (the so-called geodetic-like blocks) were used to estimate multiband delays, which are predominantly introduced by residual tropospheric delays and clock errors.

Data calibration was performed using the Astronomical Imaging System (AIPS; Greisen 2003), using a ParselTongue scripting interface (Kettenis et al. 2006). We used procedures that have been widely applied to perform high-frequency astrometry as part of the Bar and Spiral Structure Legacy (BeSSeL) Survey at $22 \mathrm{GHz}$ (Reid et al. 2009). Given the $300 \mathrm{MHz}$ frequency separation between the $v=2$ and $v=1$ transitions, we derived independent delay and rate solutions for each IF band instead of combining them. Also, since the detected maser emission in V838 Mon is relatively weak ( $<1 \mathrm{Jy}$; Sect. 3), the fringe-fitting solutions were derived from the scans on a phase-reference calibrator and then applied to the target. Phase solutions from scans on J0656-0323 were found to have a higher signal-to-noise ratio $(\mathrm{S} / \mathrm{N})$ than the solutions from J0709-0255. We therefore only used the data from J0656-0323 for the phase-referencing process.

After calibrating the visibility data, individual channel maps of $2048 \times 2048$ pixels of $60 \mu$ as were constructed using the AIPS task IMAGR. We imaged all spectral channels covering a range in local standard of rest (LSR) velocity of -1 to $110 \mathrm{~km} \mathrm{~s}^{-1}$.

\subsection{ALMA observations}

V838 Mon was observed by ALMA in band 6 with four spectral ranges: 215.56-217.44, 219.06-220.94, 230.11-231.99, and 232.06-233.94 GHz (PI: T. Kamiński). The observations took place on June 20, 2019 with the longest ALMA baselines of $15.3 \mathrm{~km}$, which resulted in an angular resolution of 22 mas (when imaged with Briggs weighting and with the robust parameter of 0.5$)$. Visibilities were calibrated with the default calibration scripts delivered by the observatory and were imaged with the Common Astronomy Software Applications package (CASA; McMullin et al. 2007). The ALMA data will be presented and analyzed in depth in a dedicated paper. Here, we present only maps of dust continuum emission and the $\mathrm{SiO} v=0, J=5-4$ line in both of which the source is clearly resolved. 


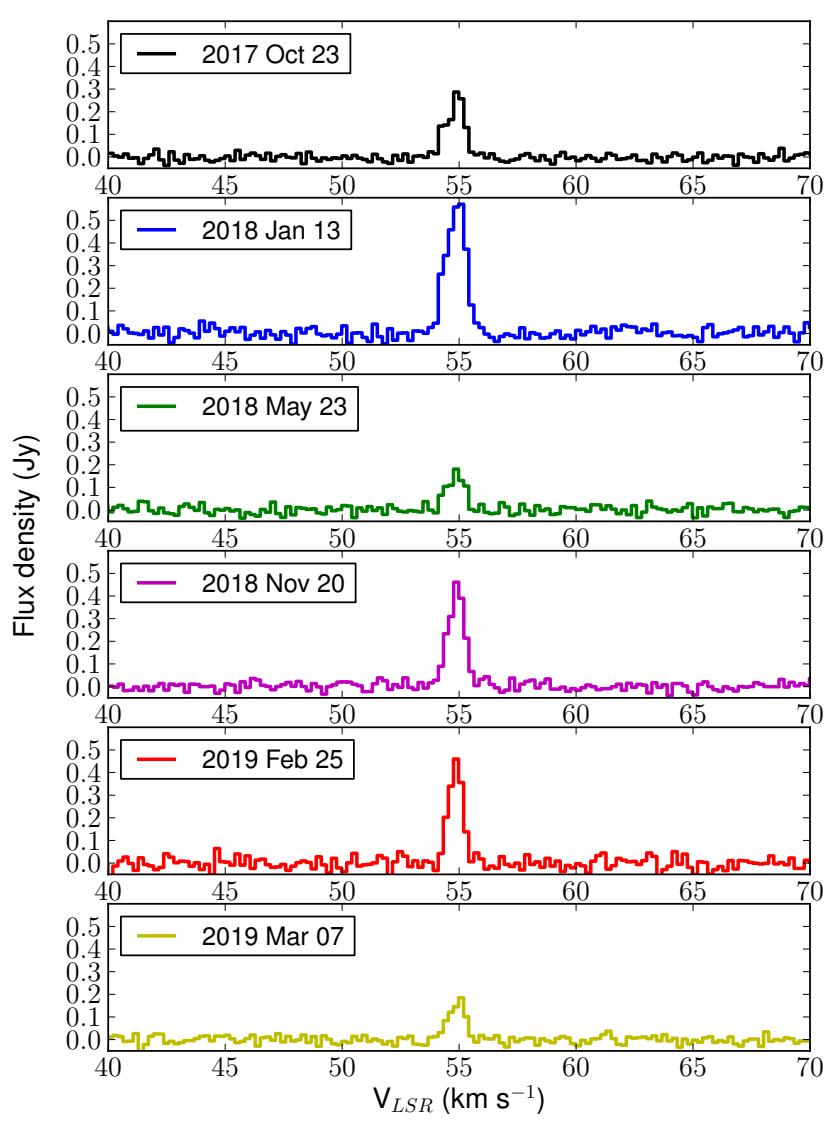

Fig. 1. Integrated flux density spectra of the $\mathrm{SiO}$ maser $(v=1, J=1-0)$ detected with the VLBA at the epoch indicated by the label in each panel.

\section{Results}

We detected maser emission from the $v=1, J=1-0$ transition at 6 epochs at LSR velocities from $\approx 54$ to $58 \mathrm{~km} \mathrm{~s}^{-1}$ with a peak near $55 \mathrm{~km} \mathrm{~s}^{-1}$ (Figs. 1 and 2). Poor observing conditions on August 27, 2018 degraded the sensitivity of the array, which resulted in a nondetection of the $v=1$ line. Integrated flux density spectra of the maser emission are shown in Fig. 1.

The $v=2, J=1-0$ transition was marginally detected only on January 13, 2018, with an integrated flux density of $0.10 \pm$ $0.03 \mathrm{Jy}$ at $55.3 \mathrm{~km} \mathrm{~s}^{-1}$. This line was not detected in the last 5 epochs.

The peak flux density of the $v=1$ transition, detected with Effelsberg in January, 2017, was $1.2 \mathrm{Jy}$, that is, almost two times stronger than we see with the VLBA. This variation in the maser intensity is more pronounced than observed before (Claussen et al. 2007). In order to investigate this in more detail, we performed a phase self-calibration, using the velocity channel with the peak flux. The flux density measured in the resulting maps from all epochs after three iterations of self-calibration was $0.7-1.2 \mathrm{Jy}$. Thus, we are detecting lower flux density in the nonself-calibrated cubes owing to residual phase errors. Other contributions to the discrepancy in flux density when compared to Effelsberg may come from large-scale emission that is resolved out by the VLBA (see Claussen et al. 2007) and the overall decline in strength that started in early 2006. Since the absolute positions are lost by self-calibrating the data, we do not use the self-calibrated images constructed for this analysis. We note that no additional maser components were found, which suggests those may be too weak to be detected with the current sensitivity of our observations.

We detected three maser features in total, where a "feature" refers to emission observed in contiguous velocity channels at nearly the same position. Channel maps using the entire $u v$ range are presented in Fig. 2 for the strongest maser feature. Unlike Claussen et al. (2007), who did not resolve emission in the peak of the line, our new VLBA maps appear to show some resolution. Figure 3 shows the emission from the second strongest feature, which is detected at low $\mathrm{S} / \mathrm{N}$ and in only four epochs at $\approx 7$ mas northeast of the strongest feature. This feature is blueshifted by $\approx 0.9 \mathrm{~km} \mathrm{~s}^{-1}$ relative to the main feature. Last, a third feature is redshifted $\left(V_{\mathrm{LSR}} \approx 58 \mathrm{~km} \mathrm{~s}^{-1}\right.$ ) and detected only in the first and second epochs at $\approx 1.5$ mas eastward of the strongest feature (Fig. 4). Figure 5 shows the spatial location of the three maser features.

\section{Astrometry}

In order to derive astrometric parameters for V838 Mon, we first selected two contiguous velocity channels with the strongest $\mathrm{SiO}$ emission (maser channels at 55.1 and $54.9 \mathrm{~km} \mathrm{~s}^{-1}$ ). The emission in these channels is resolved in most epochs, so in order to minimize the effects of extended emission we imaged the data with a minimum $u v$ length of 100 or $150 \mathrm{M} \lambda$ (the limit of $100 \mathrm{M} \lambda$ was chosen in the epochs where the maser emission is weak, since omitting the more of the data resulted in nondetections). We then fit a 2D Gaussian to the brightness distribution using the AIPS task JMFIT. The positions measured in the maps, derived with J0656-0323 as the reference source, are given in Table 2. Then, we simultaneously fit the positions of the two maser channels, solving for a single parallax $(\varpi)$, but allowing for different proper motions $\left(\mu_{\alpha} \cos \delta, \mu_{\delta}\right)$ and position offsets $\left(\alpha_{0}, \delta_{0}\right)$ at the reference epoch equal to the mean of the observed epochs. We should note that the B3V companion is between 28 and 250 au from the primary, which implies an orbital period in the range 40-1000 yr (assuming a circular orbit). Thus, we do not expect that the orbital motion has a measurable effect on the motion of the primary over the $1.4 \mathrm{yr}$ of our observations. Figure 6 shows the best fit for the resulting astrometric elements given in Table 3 . Our fitting routine computes additional systematic errors to be added in quadrature to the statistical source position errors provided by JMFIT. These errors are estimated numerically so as to make the reduced $\chi^{2} \approx 1$ for each coordinate. The resulting best fit parallax is

$\varpi=0.166 \pm 0.060 \mathrm{mas}$,

where we have multiplied the parallax uncertainty by $\sqrt{2}$ to account for possible systematic errors, which would be fully correlated for the two maser channels. Fitting each spot separately gives similar results for all astrometric parameters, but with slightly larger uncertainties.

We note that the fractional parallax error is significantly large, thus, we should take particular care when estimating the distance and its uncertainty from the parallax. Since simply inverting the parallax yields a poor approximation of the distance, we used Bayesian inference. For a truncated uniform prior, an unnormalized posterior probability density function (PDF) given by Bailer-Jones (2015) is as follows:

$P^{*}\left(r \mid \varpi, \sigma_{\varpi}\right)= \begin{cases}\frac{1}{r_{\lim }} \frac{1}{\sqrt{2 \pi} \sigma_{\varpi}} \exp \left[-\frac{1}{2 \sigma_{\varpi}^{2}}\left(\varpi-\frac{1}{r}\right)^{2}\right] & \text { if } r<0 \leq r_{\lim } \\ 0 & \text { otherwise. }\end{cases}$ 

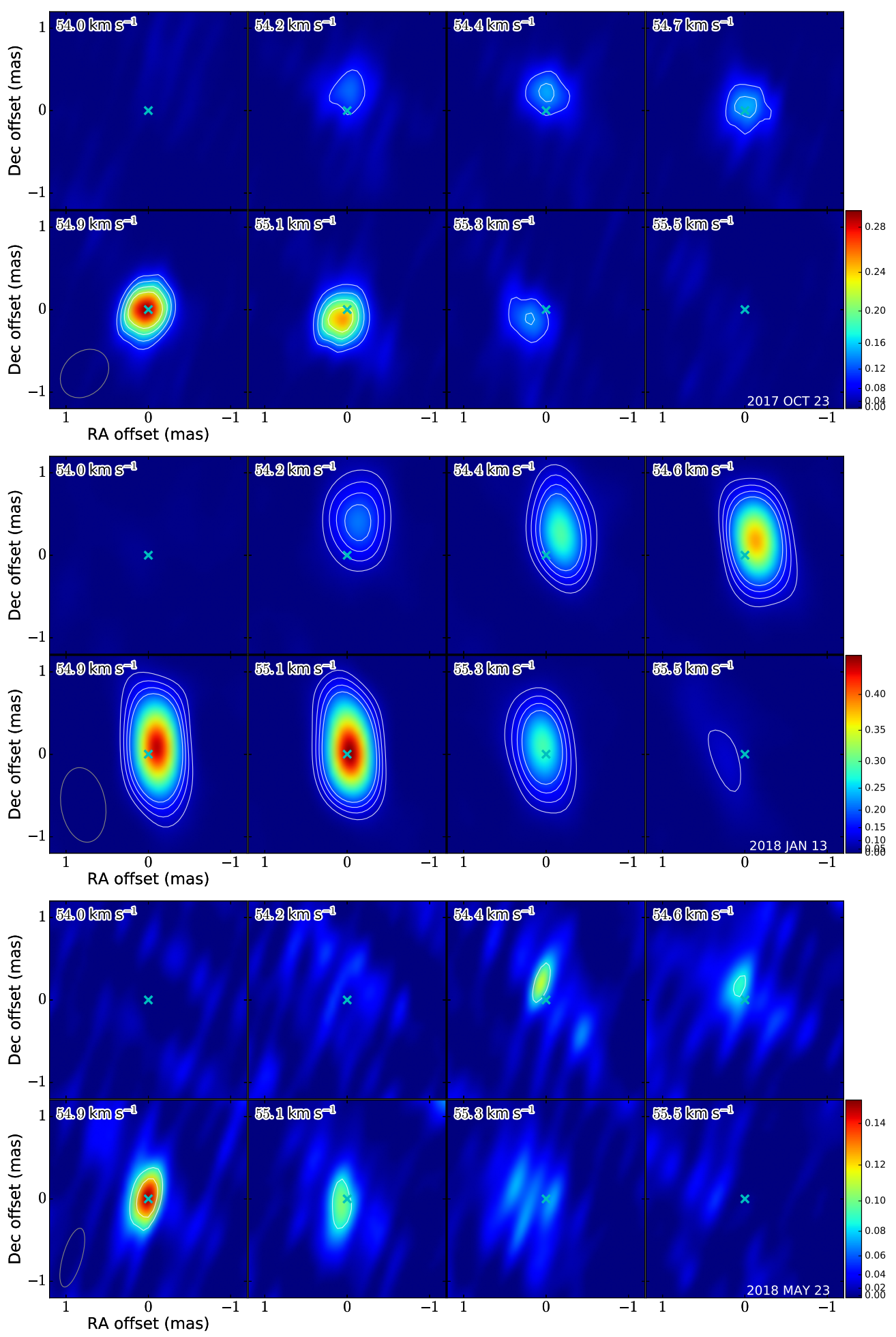

Fig. 2. Channel velocity map $\left(\mathrm{Jy} \mathrm{beam}^{-1}\right)$ of the $\mathrm{SiO}$ maser $(v=1, J=1-0)$ emission for the epoch as indicated by the label in the last panel. The contour levels are at $5 \sigma, 7 \sigma, 9 \sigma$, and $11 \sigma$, where $\sigma$ is the rms noise measured in the strongest channel of the image cube (Table 1). Position offsets are relative to the position of the strongest pixel in the channel that has the maximum peak flux (cyan cross mark).

This astrometric-based posterior PDF for $r_{\text {lim }}=20 \mathrm{kpc}$ is shown in the right panel of Fig. 7.

Finally, we apply the parallax-based distance estimator of Reid et al. (2016, 2019) for sources associated with spiral arms in the Milky Way in order to derive a combined PDF. This estimator combines other distance information including spiral arm assignment (SA), kinematic distances based on radial velocity
(KD), and two components of proper motion (PM(long) and PM(lat)):

$\operatorname{Prob}(d) \propto \operatorname{Prob}_{\mathrm{SA}}(d) \times \operatorname{Prob}_{\mathrm{KD}}(d) \times \operatorname{Prob}_{\mathrm{PM}(\text { long })}(d) \times \operatorname{Prob}_{\mathrm{PM}(\text { lat })}(d)$.

The left panel in Fig. 7 shows the individual parameters of the parallax-based distance estimator, as well as the combined PDF. 

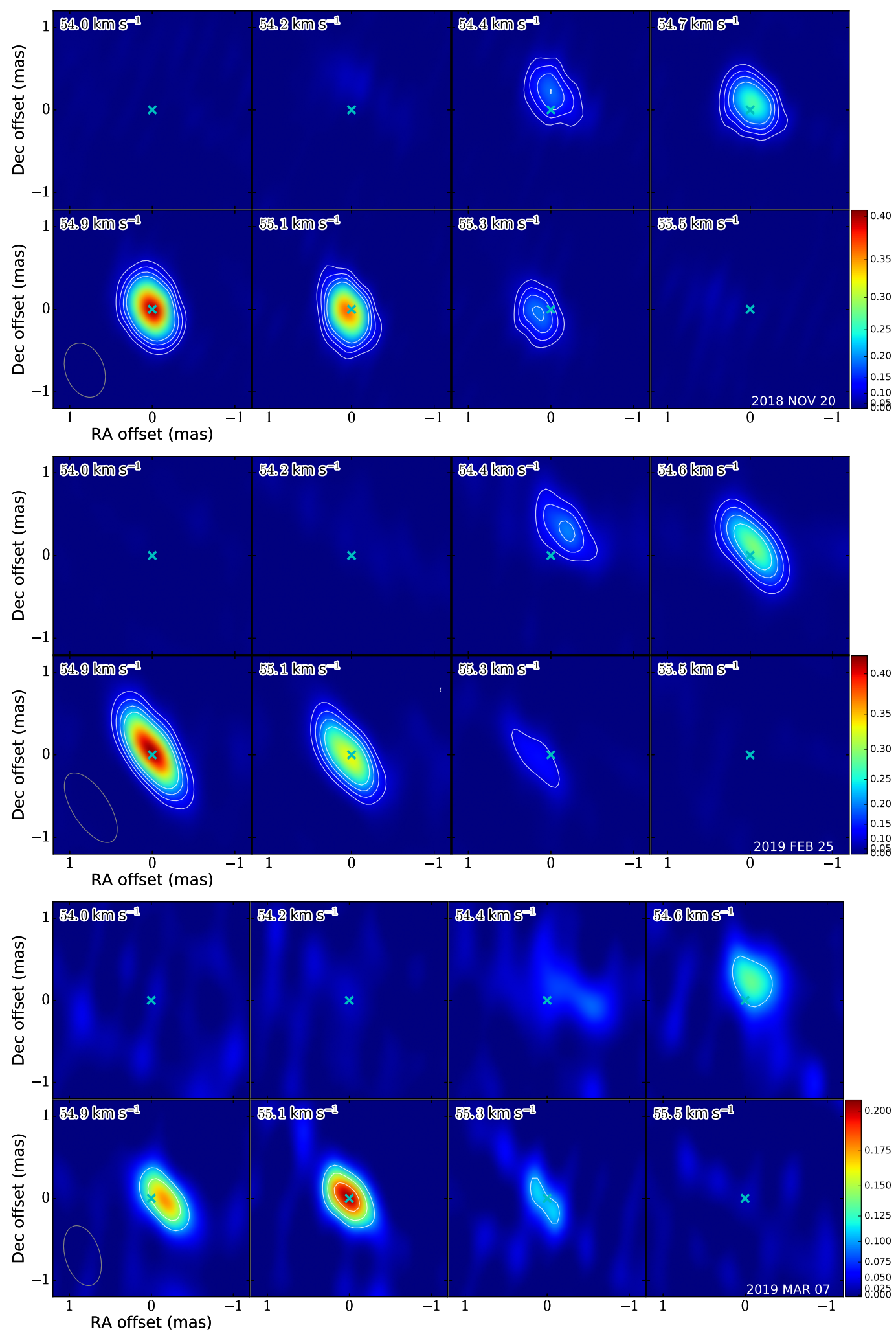

Fig. 2. continued

We have used $V_{\mathrm{LSR}}=55 \pm 5 \mathrm{~km} \mathrm{~s}^{-1}$ and the average of the proper motions of the two maser spots, $\mu_{\alpha} \cos \delta=-0.465 \pm$

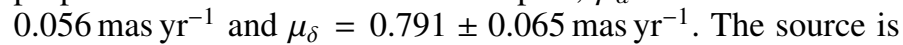
most likely associated with the Outer spiral arm at a distance of $5.5 \mathrm{kpc}$ (as previously suggested by Kamiński et al. 2011 and Quiroga-Nuñez et al. 2019).
For the second strongest spot, we were able to only estimate its proper motion using the positions fit to the brightness distribution at $V_{\mathrm{LSR}}$ of $54.2 \mathrm{~km} \mathrm{~s}^{-1}$ (listed in Table 3 and shown in Fig. 5).

We have searched the Gaia DR2 catalog and found a star (2MASS J07040482-0350506) within a search radius of 

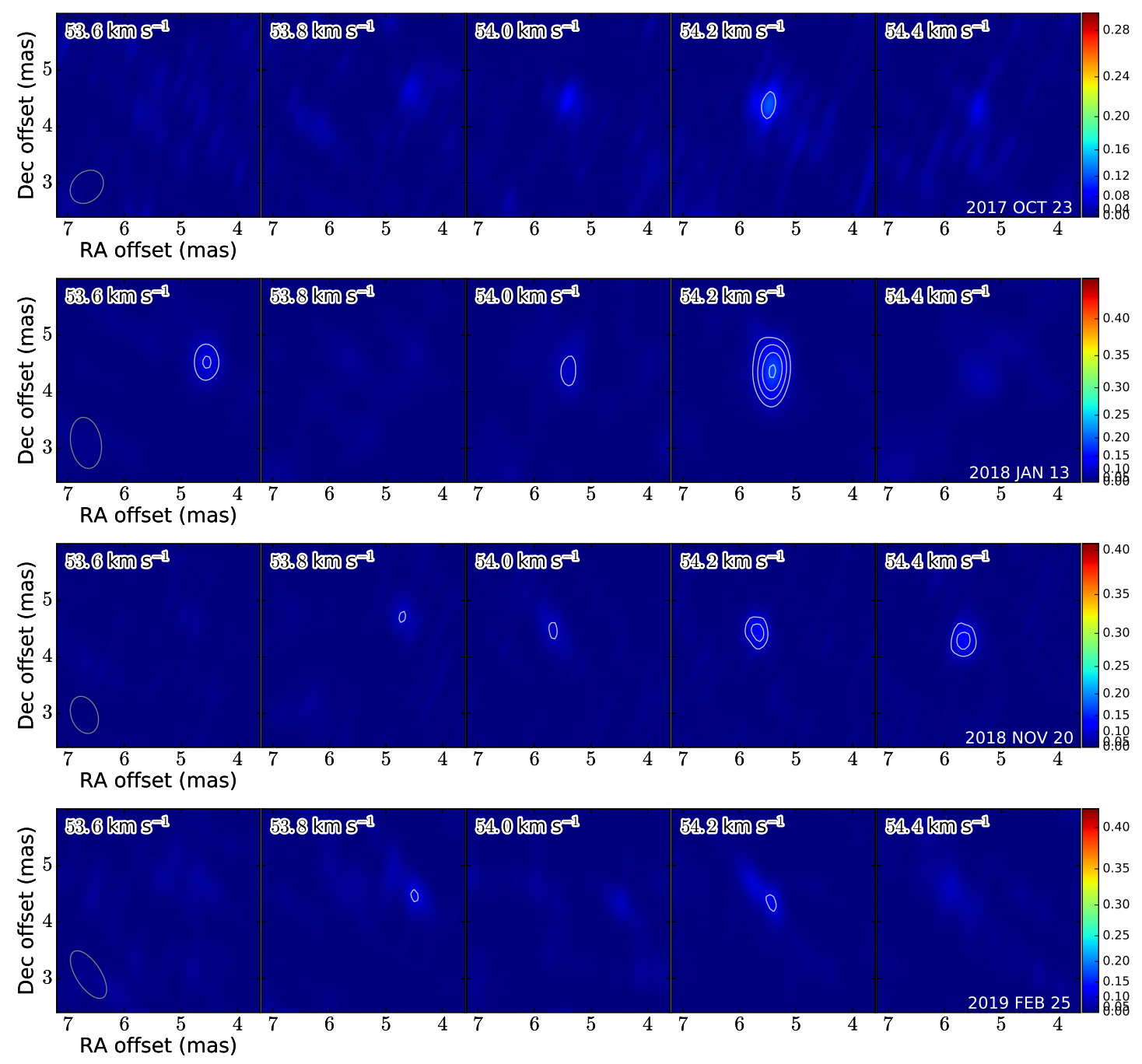

Fig. 3. Channel velocity maps $\left(\mathrm{Jy} \mathrm{beam}^{-1}\right)$ of the $\mathrm{SiO}$ maser $(v=1, J=1-0)$ emission toward the northeast of the main spot at four epochs (see dates in lower right corner). The offsets are relative to the peak position of the strongest spot in each epoch. The contour levels are at $5 \sigma, 7 \sigma, 9 \sigma$, and $11 \sigma$.

1 arcsec of the $\mathrm{SiO}$ maser position. When propagating the Gaia position at J2015.5 to the VLBA observing epochs, we found that this source has a separation of only 2.4 mas from the VLBA position of the strongest maser. This star has a DR2 parallax of $-0.001 \pm 0.105$ mas. For comparison with our VLBA distance PDF, we have constructed the Gaia DR2 distance PDF using a truncated uniform prior as shown in the right panel of Fig. 7. Similarly, we show the posterior PDF that corresponds to the light-echo distance measurement by Sparks et al. (2008). These PDFs can be combined with the distance PDF given by Eq. (3) to provide a more robust distance estimate than either alone. The combination of Eq. (3) with the VLBA astrometric-based PDF yields $d=5.5 \pm 0.6 \mathrm{kpc}$, while the combination of all PDFs yields $d=5.9 \pm 0.4 \mathrm{kpc}$, where the distance and its uncertainty have been obtained by fitting a Gaussian model to the combined PDF and estimating its peak probability density, center, and width. Table 4 summarizes all the distance results discussed above. We include the Gaia result for completeness even though it is highly uncertain and contributes little to the final distance estimate. Our study provides an example that Gaia DR2 parallaxes for distant supergiants should be treated with caution.

As described in the introduction, V838 Mon is a binary system consisting of a cool giant (the eruptive component) and the $\mathrm{B} 3 \mathrm{~V}$ companion. An effective temperature of $T_{\text {eff }} \sim$ $2000-3000 \mathrm{~K}$ was measured for the merged star from optical and IR spectroscopy in 2008-2009 (Tylenda et al. 2011; Loebman et al. 2015). The B3V companion has been attenuated by the V838 Mon outburst ejecta (Goranskij et al. 2008), which explains why it is not detected by Gaia. It is important to note that the astrometric parameters measured by Gaia toward V838 Mon may be affected by extinction from the dusty region around V838 Mon. The DR2 quality factors of the solution suggest that the source is astrometrically well-behaved; however, the relative errors on the astrometric parameters are quite large and the parallax is negative. The Gaia proper motion measurement strongly disagrees with that measured by the VLBA, as well as the Gaia proper motions of other stars in the V838 Mon cluster (see Sect. 5.1).

\section{Discussion}

\subsection{The cluster of V838 Mon}

Gaia DR2 parallaxes and proper motions of the three stars that are considered to be members of the open cluster of V838 Mon (Afşar \& Bond 2007), although highly uncertain, are 


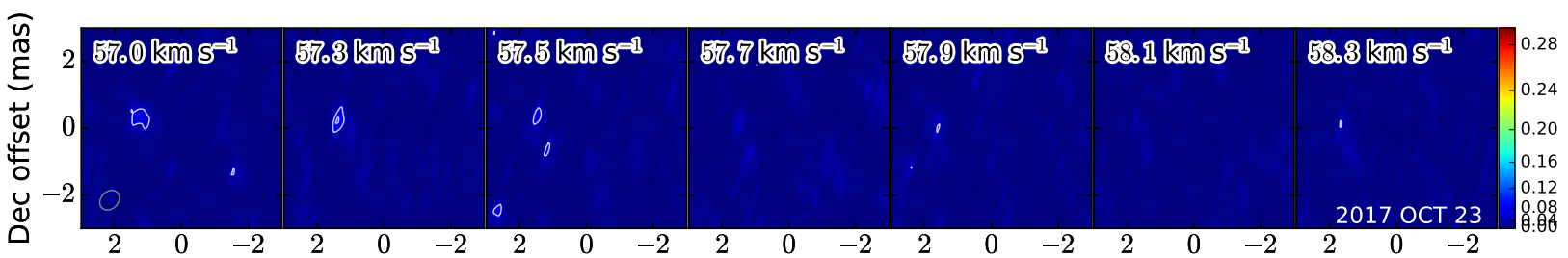

RA offset (mas)

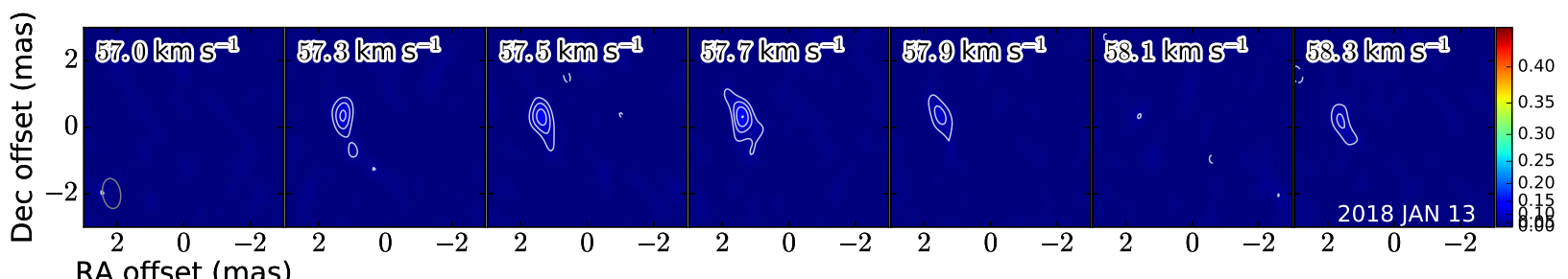

Fig. 4. Channel velocity maps $\left(\mathrm{Jy}_{\text {beam }}{ }^{-1}\right)$ of the $\mathrm{SiO}$ maser $(v=1, J=1-0)$ emission from the third spot. The offsets are relative to the peak position of the strongest spot in each epoch. The contour levels are at $3 \sigma, 5 \sigma, 7 \sigma$, and $9 \sigma$.

similar to those derived by our VLBA measurements. These stars are spectroscopically identified as B-type main-sequence stars. Their individual parallaxes are between 0.17 and 0.24 mas and have a weighted-mean parallax of $0.18 \pm 0.10$ mas, after correcting for a Gaia DR2 parallax zero-point shift of -0.029 mas $^{1}$, which agrees with our VLBA parallax of V838 Mon. Their mean

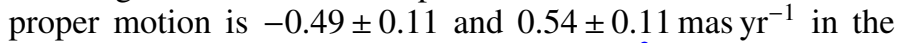
easterly and northerly directions, respectively ${ }^{2}$. We note that the mean proper motion in declination of the $\mathrm{SiO}$ masers is consistent with the mean cluster motion within $\sim 2 \sigma$ uncertainty, whereas the agreement in the parallaxes and RA proper motion is even better.

We also note that the Gaia DR2 effective temperatures for the three B-type stars with known visual spectra (Afşar \& Bond 2007) appear significantly low, most likely owing to a high extinction $\left(E_{B-V} \approx 0.8\right)$ toward the cluster not taken into account in the Gaia catalog (Andrae et al. 2018). This puts into question the Gaia effective temperature listed at $3342 \mathrm{~K}$ for V838 Mon itself, although this value is close, within $3 \sigma$, to that expected (Loebman et al. 2015).

We looked for other potential members of the cluster by searching for sources with similar parallaxes and proper motions as V838 Mon and the three cluster members. Within a radius of 2:3 of V838 Mon, we find only one candidate in the Gaia DR2 catalog, star 3107789583219504000, which is 6" east of V838 Mon. The Gaia parallax for this star $(0.16 \pm 0.14$ mas $)$ is highly uncertain, but consistent with a distance beyond $3 \mathrm{kpc}$. Its location and proper motion are shown in Fig. 8. Its angular distance from V838 Mon is the smallest among the four stars in the cluster. It is also fainter than the three B-type stars which may be indicative of a spectral type later than B6. In the maps of Wisniewski et al. (2003), this star is numbered 4 and has a slightly smaller interstellar linear polarization degree and a slightly different orientation of the polarization plane than the three confirmed cluster members. However, given the patchy morphology of the echo material and the patchy dust distribution toward the V838 Mon cluster (Sparks et al. 2008;

\footnotetext{
1 We note that larger values for the zero-point offset have been reported in the literature (e.g., -0.08 mas by Stassun \& Torres 2018). Here and throughout the paper we use the value of -0.029 mas derived by the Gaia DR2 team (Lindegren et al. 2018).

2 We have added quadratically systematic errors of 0.1 mas for parallax and $0.1 \mathrm{mas}^{-1}$ for proper motions (Luri et al. 2018).
}

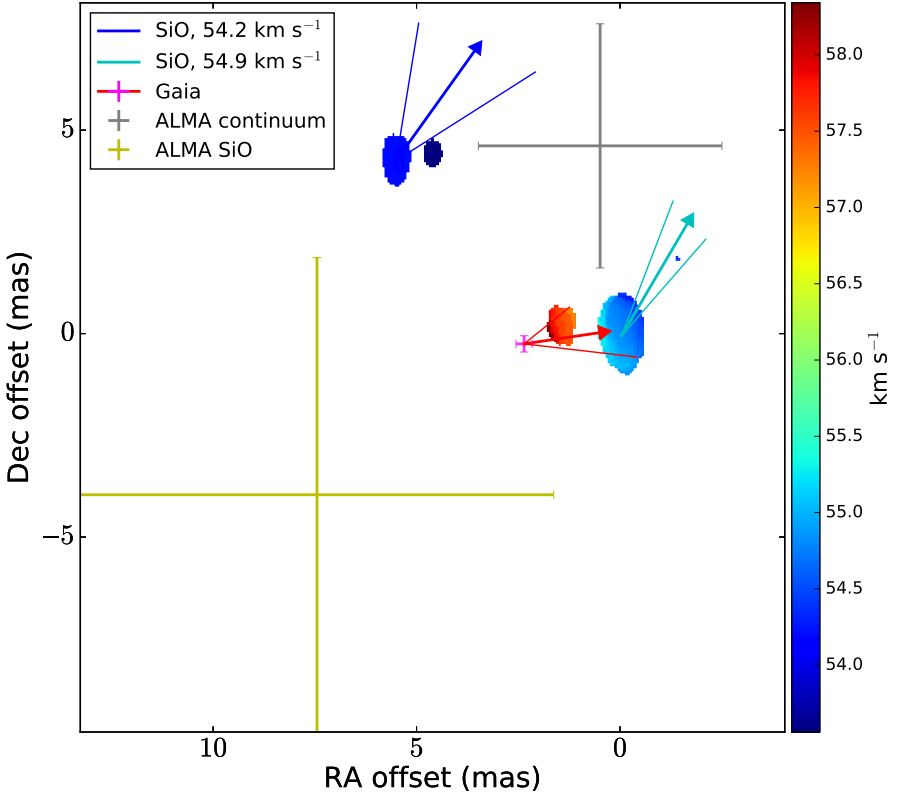

Fig. 5. Location of the spots detected on January 13, 2018 for the $v=1$, $J=1-0 \mathrm{SiO}$ maser transition. The proper motion vectors of the two strongest spots (at 54.9 and $54.2 \mathrm{~km} \mathrm{~s}^{-1}$ ) are indicated by the cyan and blue arrows, respectively. A third spot is detected at $\sim 58 \mathrm{~km} \mathrm{~s}^{-1}$ and located at $\sim 1$ mas to the east of the strongest spot. The location of the Gaia source and its proper motion is indicated by the pink cross and the red arrow, respectively. The lines associated to the arrows indicate the proper motion errors. The gray and yellow crosses mark the positions and uncertainties of the continuum and $\mathrm{SiO}$ extended emission peaks detected in the ALMA maps, respectively.

Tylenda \& Kamiński 2012), the difference does not exclude that we have identified a genuine fourth member of the cluster (not counting the stars in V838 Mon itself).

Given that the considered merger of V838 Mon took place in a triple or higher system, it is worth considering whether any star (including V838 Mon itself) could have been "kicked out" (or dynamically ejected) off the system (see e.g., Perets \& Šubr 2012). This would probably be manifested by proper motions significantly different than that of the cluster. This is not observed for V838 Mon at the current accuracy of the VLBA measurements. Indeed, after subtracting the mean proper motion 
Table 2. Source properties.

\begin{tabular}{lcccccc}
\hline \hline JD & $\begin{array}{c}\alpha \\
07^{\mathrm{h}} 04^{\mathrm{m}}\end{array}$ & $\begin{array}{c}\sigma_{\alpha} \\
(\mathrm{s})\end{array}$ & $\begin{array}{c}\delta \\
-03^{\circ} 50^{\prime}\end{array}$ & $\begin{array}{c}\sigma_{\delta} \\
(\operatorname{arcsec})\end{array}$ & $\begin{array}{c}\text { Peak flux } \\
\left(\mathrm{Jy} \mathrm{beam}^{-1}\right)\end{array}$ & $\begin{array}{c}\text { Integrated flux } \\
(\mathrm{Jy})\end{array}$ \\
\hline \multicolumn{2}{l}{$\begin{array}{l}\text { Maser } V_{\mathrm{LSR}}=55.1 \mathrm{~km} \mathrm{~s}^{-1} \\
2458049.99042\end{array}$} & & & & & \\
2458131.76652 & 4.8215052 & $1.0 \mathrm{e}-06$ & 50.634242 & $2.8 \mathrm{e}-05$ & $0.22 \pm 0.02$ & $0.24 \pm 0.04$ \\
2458262.40884 & 4.8214917 & $2.2 \mathrm{e}-06$ & 50.633577 & $7.3 \mathrm{e}-05$ & $0.10 \pm 0.02$ & $0.29 \pm 0.06$ \\
2458442.91462 & 4.8214881 & $6.3 \mathrm{e}-07$ & 50.633402 & $2.0 \mathrm{e}-05$ & $0.22 \pm 0.02$ & $0.32 \pm 0.04$ \\
2458539.64977 & 4.8214540 & $1.5 \mathrm{e}-06$ & 50.633266 & $2.9 \mathrm{e}-05$ & $0.26 \pm 0.02$ & $0.30 \pm 0.04$ \\
2458550.61973 & 4.8214685 & $1.5 \mathrm{e}-06$ & 50.633057 & $2.7 \mathrm{e}-05$ & $0.22 \pm 0.02$ & $0.25 \pm 0.04$ \\
\hline Maser $V_{\mathrm{LSR}}=54.9 \mathrm{~km} \mathrm{~s}^{-1}$ & & & & & \\
2458049.99042 & 4.8215162 & $8.9 \mathrm{e}-07$ & 50.634123 & $2.7 \mathrm{e}-05$ & $0.18 \pm 0.02$ & $0.29 \pm 0.05$ \\
2458131.76652 & 4.8215011 & $1.0 \mathrm{e}-06$ & 50.634140 & $3.2 \mathrm{e}-05$ & $0.22 \pm 0.02$ & $0.25 \pm 0.04$ \\
2458262.40884 & 4.8214887 & $1.8 \mathrm{e}-06$ & 50.633464 & $4.5 \mathrm{e}-05$ & $0.12 \pm 0.02$ & $0.23 \pm 0.05$ \\
2458442.91462 & 4.8214843 & $7.9 \mathrm{e}-07$ & 50.633382 & $2.2 \mathrm{e}-05$ & $0.22 \pm 0.02$ & $0.36 \pm 0.05$ \\
2458539.64977 & 4.8214532 & $1.4 \mathrm{e}-06$ & 50.633232 & $2.5 \mathrm{e}-05$ & $0.28 \pm 0.02$ & $0.35 \pm 0.05$ \\
2458550.61973 & 4.8214566 & $1.9 \mathrm{e}-06$ & 50.633088 & $3.4 \mathrm{e}-05$ & $0.19 \pm 0.02$ & $0.23 \pm 0.04$ \\
\hline
\end{tabular}
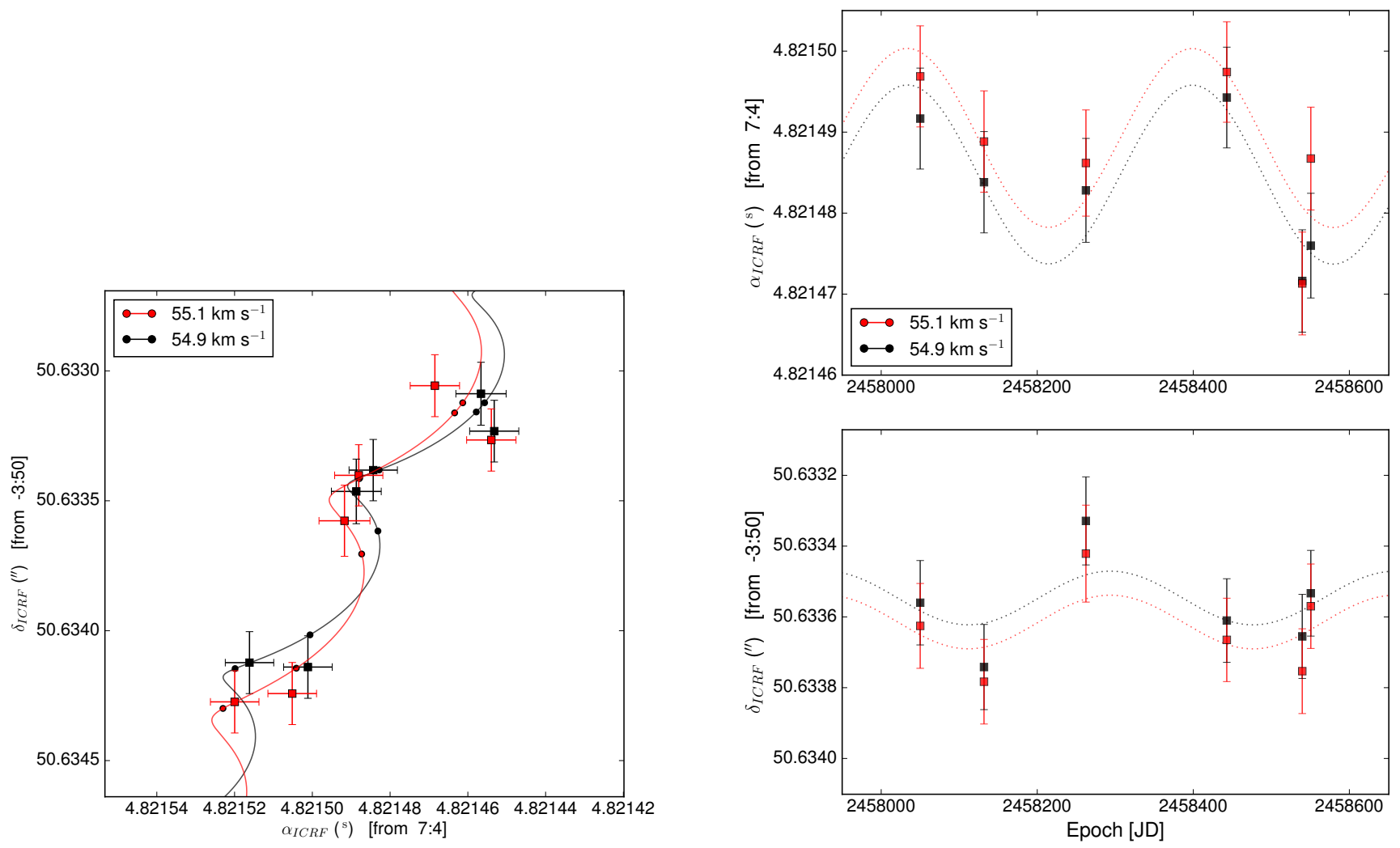

Fig. 6. Left: measured VLBA sky positions of V838 Mon maser spots in two contiguous channels (black and red squares). The line corresponds to the best fit to the data while the filled circles are the expected positions from this fit at the observed epochs. Rigth: right ascension (upper panel) and declination (lower panel) of the maser spots as a function of time after subtracting the fit proper motions to see only the effect of the parallax.

of the cluster from the VLBA proper motions, we obtain that V838 Mon has a transverse velocity relative to the cluster of $7 \pm 5 \mathrm{~km} \mathrm{~s}^{-1}$. The radial component is unknown. This low velocity implies a small displacement of V838 Mon as a result of the "kick". If we assume that the ejection took place in 2002, we obtain a displacement of only $4 \pm 2$ mas or $25 \pm 12 \mathrm{au}$. The low velocity is inconsistent with the ejection scenario. However, constraints on the motion of the now dust-embedded physical companion of V838 Mon are very poor (Kamiński et al. 2009), thus we cannot completely rule out that this component has been dynamically ejected.

\subsection{Location of the stellar photosphere in VLBA maps}

Optical observations, the photospheric size (Chesneau et al. 2014), and the presence of an $\mathrm{SiO}$ maser make V838 Mon very similar to red supergiants with highest mass-loss rates. $\mathrm{SiO}$ masers observed in Asymptotic Giant Branch (AGB) stars, and 
Table 3. Astrometric parameters from VLBA.

\begin{tabular}{lcccccc}
\hline \hline $\begin{array}{l}\text { Maser } V_{\mathrm{LSR}} \\
\left(\mathrm{km} \mathrm{s}^{-1}\right)\end{array}$ & $\begin{array}{c}\alpha_{0} \\
07^{\mathrm{h}} 04^{\mathrm{m}}\end{array}$ & $\begin{array}{c}\delta_{0} \\
-03^{\circ} 50^{\prime}\end{array}$ & $\begin{array}{c}\mu_{\alpha} \cos \delta \\
\left(\mathrm{mas} \mathrm{yr}^{-1}\right)\end{array}$ & $\begin{array}{c}\mu_{\delta} \\
\left(\mathrm{mas} \mathrm{yr}^{-1}\right)\end{array}$ & $\begin{array}{c}\varpi \\
(\mathrm{mas})\end{array}$ & $\begin{array}{c}\mathrm{D}^{(a)} \\
(\mathrm{kpc})\end{array}$ \\
\hline 55.1 & $4.82149(7)$ & $500^{\prime \prime} 6336(1)$ & $-0.451 \pm 0.079$ & $0.847 \pm 0.092$ & $0.166 \pm 0.060$ & $6.0_{-1.6}^{+3.4}$ \\
54.9 & $4.82148(7)$ & $50^{\prime \prime} 6335(1)$ & $-0.479 \pm 0.079$ & $0.736 \pm 0.092$ & & - \\
Average & - & - & $-0.465 \pm 0.056$ & $0.791 \pm 0.065$ & - & - \\
\hline 54.2 & $4.82186(2)$ & $50.6293(1)$ & $-0.553 \pm 0.339$ & $0.771 \pm 0.167$ & - & - \\
\hline
\end{tabular}

Notes. Numbers in parenthesis give position errors in units of the last significant digits. ${ }^{(a)}$ Distance inferred from the inversion of the parallax.
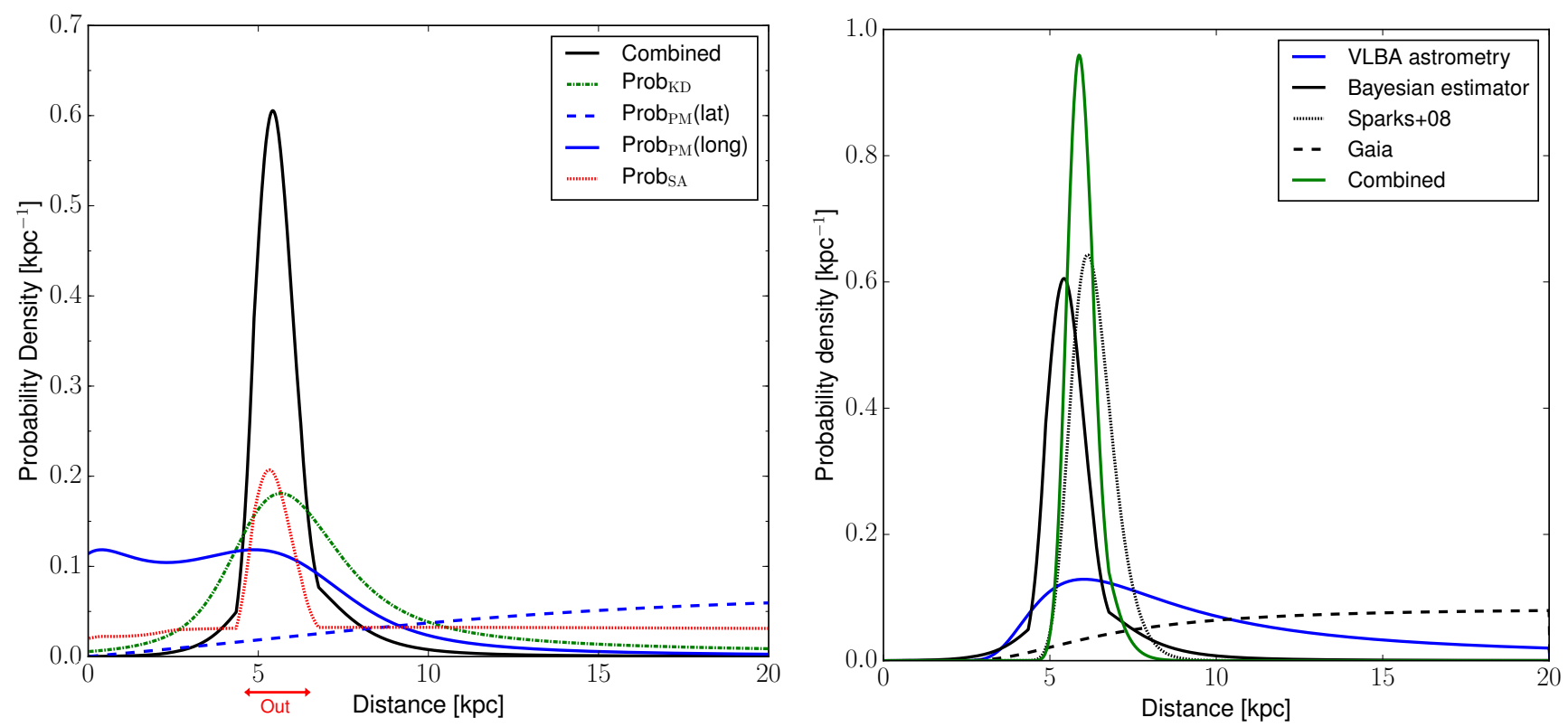

Fig. 7. Left: normalized distance PDF for V838 Mon (black line) which combines kinematic distance (green dashed line), Galactic latitude and spiral arm model (red dashed line), and source's proper motion in Galactic longitude (blue solid line) and Galactic latitude (blue dashed line). The red arrow marks the distance range for possible arm associations. Right: normalized distance PDF for the various measurements of the V838 Mon parallax, including our VLBA astrometry, the Bayesian estimator, Gaia DR2, and the light echo.

Table 4. Parallax measurements.

\begin{tabular}{lccc}
\hline \hline$\varpi[\mathrm{mas}]$ & & Instrument & Reference \\
\hline$-0.001 \pm 0.105$ & & Gaia astrometry & 1,2 \\
$0.166 \pm 0.060$ & & VLBA astrometry & 3 \\
\hline$\varpi[\mathrm{mas}]$ & Distance $[\mathrm{kpc}]$ & Method & Reference \\
\hline $0.164 \pm 0.016$ & $6.1 \pm 0.6$ & Light-echo polarimetry & 4 \\
$0.182 \pm 0.019$ & $5.5 \pm 0.6$ & Distance estimator & 3 \\
$0.181 \pm 0.018$ & $5.5 \pm 0.6$ & Distance estimator+VLBA & 3 \\
$0.169 \pm 0.011$ & $5.9 \pm 0.4$ & Distance estimator+VLBA+light echo+Gaia & 3 \\
\hline
\end{tabular}

References. (1) Gaia Collaboration (2018); (2) Bailer-Jones et al. (2018); (3) this work; (4) Sparks et al. (2008).

Mira stars in particular, often take the form of a ring surrounding the star. In such cases, it is relatively straightforward to identify the location of the stellar photosphere, even if it is not directly detected in the same observations (see, e.g., Cotton et al. 2008). This task is more difficult for red supergiants whose mass loss may be very inhomogeneous and the distribution of $\mathrm{SiO}$ maser spots may be very erratic. VYCMa is the best known example of such a complexity (Zhang et al. 2012). In our maps of the maser emission in V838 Mon we probably detect only the three brightest $\mathrm{SiO}$ maser spots. Assuming that their distribution is as complex as in red supergiants, we are unable to identify the location of the stellar photosphere in the radio maps. Given that V838 Mon is an unresolved binary for Gaia, we are also unable to associate the Gaia position to the V838 Mon merger product or the companion. The stellar remnant of the component of V838 Mon that erupted in 2002 remains the brightest radiation source of the system. Part of its energy is generated by continuing contraction and thus it has a higher bolometric luminosity than the B3V companion. The stellar radiation heats the surrounding dust expelled during the eruption producing emission at millimeter wavelengths. The emission is brightest closest to the contracting luminous star. Thus, we can take the 


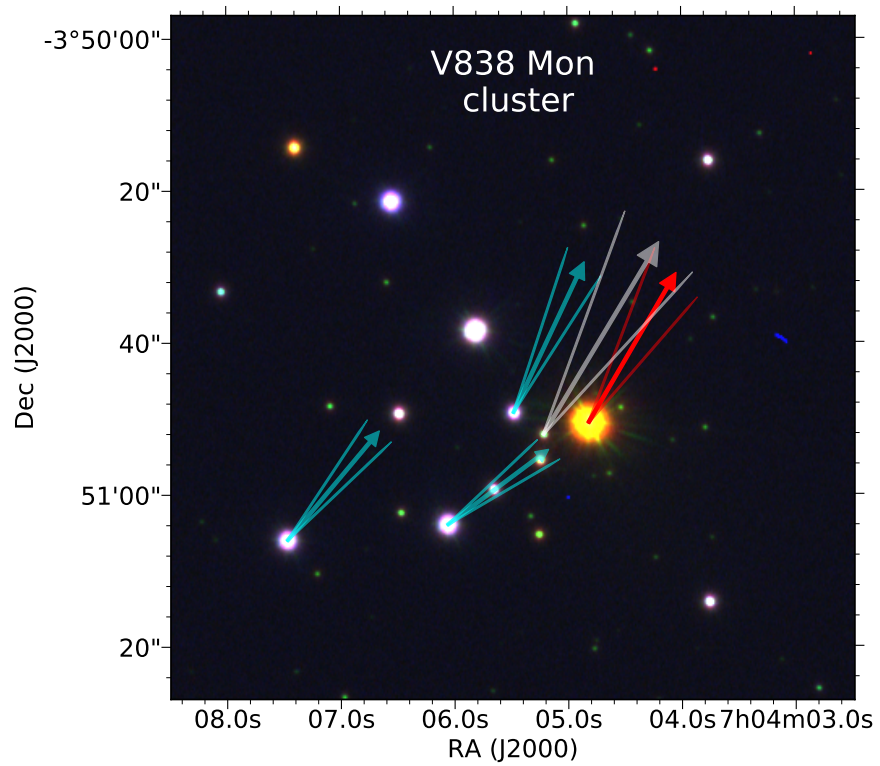

Fig. 8. Proper motions of the identified members of the young open cluster of V838 Mon. The arrows show the proper motions scaled by a factor of 25000 and the associated lines indicate the errors of the measurements. Red arrow corresponds to our VLBA measurement for V838 Mon, blue arrows correspond to Gaia DR2 values for identified cluster members, and the gray arrow is drawn for a new candidate member of the cluster and represents its proper motion in the Gaia DR2 catalog. The background image is a color rendition of images obtained in 2015 with the OmegaCAM imager mounted on the ESO's VLT Survey Telescope (Drew et al. 2014); red, green, and blue colors correspond to $r, g$, and $u$ filters, respectively.

position of the millimeter-wave continuum peak as the stellar (photosphere) position. We see in Fig. 5 that this peak is located between the two brightest maser spots (within the modest accuracy of the ALMA position measurements; $\approx \pm 3$ mas).

Chesneau et al. (2014) observed the mid-IR (dust) emission around V838 Mon with the Very Large Telescope Interferometer array and derived a major axis size of 25 mas at $8 \mu \mathrm{m}$ and 70 mas at $13 \mu \mathrm{m}$ (147 and 412 au at $5.9 \mathrm{kpc}$, respectively). The dust emission is flatter at $13 \mu \mathrm{m}$ and has a position angle of about $-10^{\circ}$. This dusty structure, interpreted as a disk, is believed to have formed after the outburst in 2002 as a result of the merger event. For the central star, Chesneau et al. (2014) derived a diameter of $1.15 \pm 0.20$ mas $(\approx 6.8$ au at $5.9 \mathrm{kpc})$. The $\mathrm{SiO}$ maser spots are located within the extended dusty structure around V838 Mon at $\approx 5$ mas $(\approx 30 \mathrm{au})$ from the stellar position (as measured in the ALMA continuum map), that is, at $\approx 9$ stellar radii but within the dusty structure identified by Chesneau et al. (2014). This supports the idea that V838 Mon and AGB stars resemble each other, since in AGBs the $\mathrm{SiO}$ masers are found in the immediate vicinity of the stellar photosphere (Cotton et al. 2008).

\subsection{Extended emission detected with ALMA}

The velocity-integrated intensity map of the SiO thermal emission detected by ALMA is shown in Fig. 9. This corresponds to the $\mathrm{SiO} v=0, J=5-4$ transition at $217 \mathrm{GHz}$, which shows a very broad profile with a FWHM of $225 \mathrm{~km} \mathrm{~s}^{-1}$ for the entire emission region. The emission has an extended structure with a beam-deconvolved size of $148 \times 125( \pm 30)$ mas. The major axis of this structure is aligned at a position angle of $61 \pm 7^{\circ}$. Continuum emission contours at $225 \mathrm{GHz}$ are overlaid in Fig. 9. The continuum has a peak flux density of $0.43 \mathrm{mJy}$ beam $^{-1}$ and an elongated morphology in a direction almost perpendicular to the major axis of the $\mathrm{SiO}$ thermal emission. The extent of the continuum structure is about 150 mas. Gaussian fits yield the continuum peak at RA = 07:04:04.821535 $( \pm 0.17 \mathrm{mas})$ and $\mathrm{Dec}=-03: 50: 50.629426( \pm 0.18 \mathrm{mas})$, and the extended $\mathrm{SiO}$ emission peaks at $\mathrm{RA}=07: 04: 04.822( \pm 5$ mas) and Dec $=-03: 50: 50.638( \pm 5$ mas) (ICRS), where the errors express only formal uncertainties of the centroiding procedure. The astrometric accuracy of the ALMA observations is $\approx 3$ mas. ${ }^{3}$ The position of the strongest $\mathrm{SiO}$ maser spot agrees within $2 \sigma$ with the peaks of continuum and thermal $\mathrm{SiO}$ emission (see Fig. 5).

As pointed out by Kamiński et al. (2018), the millimeter emission observed with ALMA in this and other red novae is produced by material from the merger ejecta. Then, it is worth noting that the elongated morphology resembles that of a jet. The formation of jets during a merger event has been discussed in Kashi \& Soker (2016), for example. An elongated morphology of the merger ejecta is also consistent with mass loss in the orbital plane of the merging stars (e.g., Pejcha et al. 2016). This requires the orbit to be almost edge-on. However, the inclination of the orbit, as well as the rest of the orbital parameters, has not been yet constrained. The morphology of the millimeter continuum, which traces dust, is different than that of the $\mathrm{SiO}$, which is tracing the outflow. However, other lines like ${ }^{12} \mathrm{CO},{ }^{13} \mathrm{CO}$, $\mathrm{AlOH}, \mathrm{SO}_{2}$, and $\mathrm{H}_{2} \mathrm{~S}$ show emission spatially coincident with the continuum. The thermal submillimeter emission of V838 Mon will be analyzed in a dedicated study.

\section{Conclusions}

Multiepoch VLBA observations of the $\operatorname{SiO} v=1,2 J=1-0$ maser transitions at $43 \mathrm{GHz}$ have been obtained toward the red transient V838 Mon. Three maser spots from the $v=1$ line were firmly detected, while the $v=2$ line was tentatively detected in only one epoch. We modeled the motion of maser spots which were detected in two contiguous channels at 6 epochs to fit the astrometric parameters of the source. The resulting best-fit parallax is $0.166 \pm 0.060$ mas. Using Bayesian inference, we combined our VLBA parallax with other distance information to arrive at an improved distance of $5.9 \pm 0.4 \mathrm{kpc}$. Quiroga-Nuñez et al. (2019) and Reid et al. (2019) considered whether V838 Mon is in the Perseus arm, the Outer arm, or the interam region, and favored the Outer arm. The improved distance of $5.9 \pm 0.4 \mathrm{kpc}$ indicates that it is further than $5 \mathrm{kpc}$ which favors it being in the Outer arm.

Proper motions of field stars in the vicinity of V838 Mon were taken from Gaia DR2. The mean proper motion of the three known members of the V838 Mon open cluster is consistent within $2 \sigma$ with the VLBA proper motions, confirming its membership. A possible new member of the cluster was also identified.

Finally, observations taken with ALMA of $\mathrm{SiO}$ thermal emission at $217 \mathrm{GHz}$ revealed an extended outflow with a size of $148 \times 125$ mas $(870 \times 740 \mathrm{au})$. The major axis of this structure is perpendicular to $225 \mathrm{GHz}$ continuum emission, which shows an elongated and complex structure.

\footnotetext{
3 https://help.almascience.org/index. php?/Knowledgebase/Article/View/319/6/ what-is-the-astrometric-accuracy-of-alma
} 

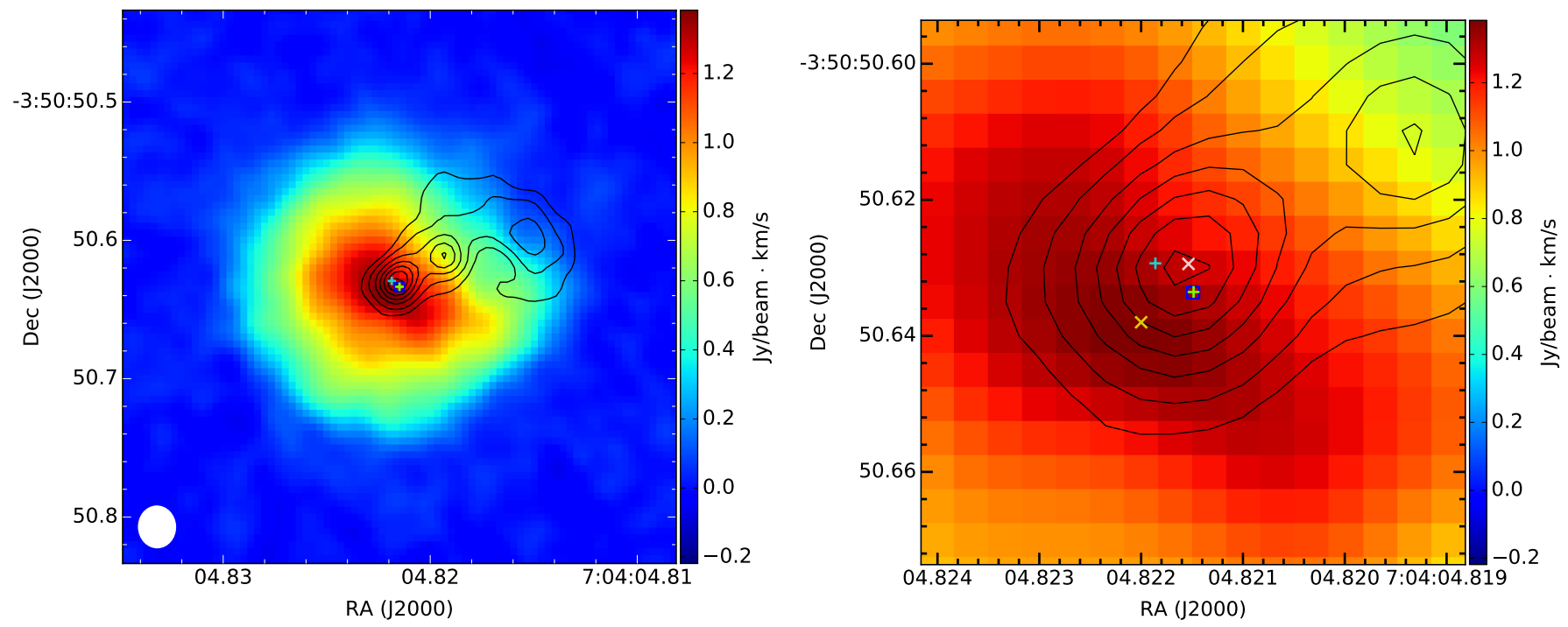

Fig. 9. Moment zero map of the $\mathrm{SiO}$ molecular emission observed with ALMA. The contours show the continuum emission at 10, 20, 35, 50, 65, 80, and $95 \%$ of the peak emission. The positions of the $\mathrm{SiO}$ masers detected with the VLBA are indicated by two the pluses and the square. Right panel: zoom-in of the central part of the map. The white and yellow crosses mark the continuum and $\mathrm{SiO}$ extended emission peaks detected in the ALMA maps, respectively.

Acknowledgements. The authors sincerely acknowledge the anonymous referee, whose comments improved the manuscript. G. N. O.-L. acknowledges support from the Alexander von Humboldt Foundation. TK acknowledges funding from Grant no 2018/30/E/ST9/00398 from the Polish National Science Centre. R. T. acknowledges a support from grant 2017/27/B/ST9/01128 financed by the Polish National Science Centre. The Long Baseline Observatory is a facility of the National Science Foundation operated under cooperative agreement by Associated Universities, Inc. The National Radio Astronomy Observatory is a facility of the National Science Foundation operated under cooperative agreement by Associated Universities, Inc. This work has made use of data from the European Space Agency (ESA) mission Gaia (https://www.cosmos.esa.int/gaia), processed by the Gaia Data Processing and Analysis Consortium (DPAC, https://www. cosmos.esa.int/ web/gaia/dpac/consortium). Funding for the DPAC has been provided by national institutions, in particular the institutions participating in the Gaia Multilateral Agreement. This paper makes use of the following ALMA data: ADS/JAO.ALMA\#2018.1.00336.S. ALMA is a partnership of ESO (representing its member states), NSF (USA) and NINS (Japan), together with NRC (Canada), MOST and ASIAA (Taiwan), and KASI (Republic of Korea), in cooperation with the Republic of Chile. The Joint ALMA Observatory is operated by ESO, AUI/NRAO, and NAOJ. The National Radio Astronomy Observatory is a facility of the National Science Foundation operated under cooperative agreement by Associated Universities, Inc. Based on observations collected at the European Organisation for Astronomical Research in the Southern Hemisphere under ESO programme 177.D-3023(F).

\section{References}

Afşar, M., \& Bond, H. E. 2007, AJ, 133, 387

Andrae, R., Fouesneau, M., Creevey, O., et al. 2018, A\&A, 616, A8

Bailer-Jones, C. A. L. 2015, PASP, 127, 994

Bailer-Jones, C. A. L., Rybizki, J., Fouesneau, M., Mantelet, G., \& Andrae, R. 2018, AJ, 156, 58

Bond, H. E., Henden, A., Levay, Z. G., et al. 2003, Nature, 422, 405 Chesneau, O., Millour, F., De Marco, O., et al. 2014, A\&A, 569, L3

Claussen, M. J., Bond, H. E., Starrfield, S., \& Healy, K. 2007, in The Nature of V838 Mon and its Light Echo, eds. R. L. M. Corradi, \& U. Munari, ASP Conf. Ser., 363, 87

Cotton, W. D., Perrin, G., \& Lopez, B. 2008, A\&A, 477, 853

Crause, L. A., Lawson, W. A., Kilkenny, D., et al. 2003, MNRAS, 341, 785

Deguchi, S., Matsunaga, N., \& Fukushi, H. 2005, PASJ, 57, L25

Deguchi, S., Matsunaga, N., Fukushi, H., \& Kaminski, T. 2009, ATel, 1996
Drew, J. E., Gonzalez-Solares, E., Greimel, R., et al. 2014, MNRAS, 440, 2036 Gaia Collaboration (Brown, A. G. A., et al.) 2018, A\&A, 616, A1 Goranskij, V., Zharova, A., Barsukova, E., Fabrika, S., \& Valeev, A. 2008, ArXiv e-prints [arXiv:0810.1887]

Greisen, E. W. 2003, ASSL, 285, 109

Kamiński, T., Schmidt, M., Tylenda, R., Konacki, M., \& Gromadzki, M. 2009, ApJS, 182, 33

Kamiński, T., Tylenda, R., \& Deguchi, S. 2011, A\&A, 529, A48

Kamiński, T., Steffen, W., Tylenda, R., et al. 2018, A\&A, 617, A129

Kashi, A., \& Soker, N. 2016, Res. Astron. Astrophys., 16, 99

Kettenis, M., van Langevelde, H. J., Reynolds, C., \& Cotton, B. 2006, in Astronomical Data Analysis Software and Systems XV, eds. C. Gabriel, C. Arviset, D. Ponz, \& S. Enrique, ASP Conf. Ser., 351, 497

Lindegren, L., Hernández, J., Bombrun, A., et al. 2018, A\&A, 616, A2

Loebman, S. R., Wisniewski, J. P., Schmidt, S. J., et al. 2015, AJ, 149, 17

Luri, X., Brown, A. G. A., Sarro, L. M., et al. 2018, A\&A, 616, A9

McMullin, J. P., Waters, B., Schiebel, D., Young, W., \& Golap, K. 2007, in CASA Architecture and Applications, eds. R. A. Shaw, F. Hill, \& D. J. Bell, ASP Conf. Ser., 376, 127

Müller, H. S. P., Spezzano, S., Bizzocchi, L., et al. 2013, J. Phys. Chem. A, 117, 13843

Munari, U., Henden, A., Kiyota, S., et al. 2002a, A\&A, 389, L51

Munari, U., Desidera, S., \& Henden, A. 2002b, IAU Circ., 8005, 2

Munari, U., Henden, A., Vallenari, A., et al. 2005, A\&A, 434, 1107

Munari, U., Corradi, R. L. M., Henden, A., et al. 2007, A\&A, 474, 585

Pejcha, O., Metzger, B. D., \& Tomida, K. 2016, MNRAS, 455, 4351

Perets, H. B., \& Subr, L. 2012, ApJ, 751, 133

Quiroga-Nuñez, L. H., Immer, K., van Langevelde, H. J., Reid, M. J., \& Burns, R. A. 2019, A\&A, 625, A70

Reid, M. J., Menten, K. M., Brunthaler, A., et al. 2009, ApJ, 693, 397

Reid, M. J., Dame, T. M., Menten, K. M., \& Brunthaler, A. 2016, ApJ, 823, 77

Reid, M. J., Menten, K. M., Brunthaler, A., et al. 2019, ApJ, 885, 131

Sparks, W. B., Bond, H. E., Cracraft, M., et al. 2008, AJ, 135, 605

Stassun, K. G., \& Torres, G. 2018, ApJ, 862, 61

Tylenda, R. 2004, A\&A, 414, 223

Tylenda, R., \& Kamiński, T. 2012, A\&A, 548, A23

Tylenda, R., \& Soker, N. 2006, A\&A, 451, 223

Tylenda, R., Kamiński, T., \& Schmidt, M. 2009, A\&A, 503, 899

Tylenda, R., Kamiński, T., Schmidt, M., Kurtev, R., \& Tomov, T. 2011, A\&A, 532, A138

Wagner, R. M., \& Starrfield, S. G. 2002, IAU Circ., 7992, 2

Wisniewski, J. P., Bjorkman, K. S., \& Magalhães, A. M. 2003, ApJ, 598, L43

Zhang, B., Reid, M. J., Menten, K. M., \& Zheng, X. W. 2012, ApJ, 744, 23 